\title{
Chamber Propagation Physics for Heavy Ion Fusion*
}

\author{
Debra A. Callahan \\ Lawrence Livermore National Laboratory, University of California \\ P.O. Box 5508, L-440, Livermore California 94550, USA
}

\section{RECENED}

\section{FEB 061996}

This paper was prepared for submittal to the $0 S 1$

Proceedings of the International Symposium On Heavy Ion Fusion

Princeton, New Jersey

September 6-9, 1995

September 1, 1995
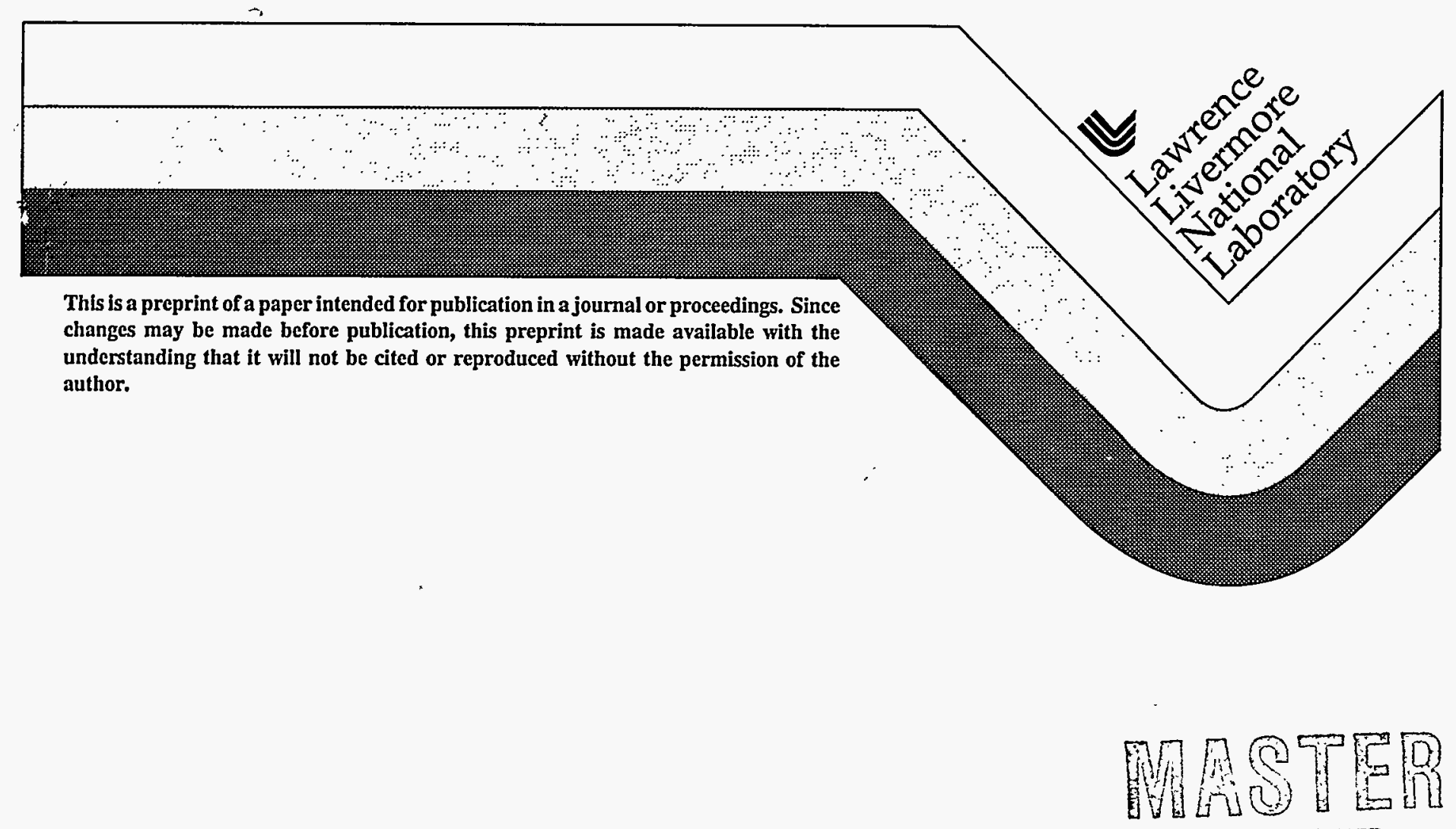


\section{DISCLAIMER}

This document was prepared as an account of worksponsored by an agency of the United States Government. Neither the United States Government nor the University of California nor any of their employees, makes any warranty, express or implied, or assumes any legal liability of responsibility for the accuracy, completeness, or usefulness of any information, apparatus, product, or process disclosed, or represents that its use would not infringe privately owned rights. Reference herein to any specific commercial products, process, or service by trade name, trademark, manufacturer, or otherwise, does not necessarily constitute or imply its endorsement, recommendation, or favoring by the United States Government or the University of Califomia. The views and opinions of authors expressed herein do not necessarily state or reflect those of the United States Government or the University of California, and shall not be used for advertising or product endorsement purposes. 


\title{
Chamber Propagation Physics for Heavy Ion Fusion*
}

\author{
Debra A. Callahan \\ Lawrence Livermore National Laboratory \\ P. O. Box $808 \mathrm{~L}-440$ \\ Livermore, CA 94551
}

September 1, 1995

\begin{abstract}
Chamber transport is an important area of study for heavy ion fusion. Final focus and chamber'transport are high leverage areas providing opportunities to significantly decrease the cost of electricity from a heavy ion fusion power plant. Chamber transport in two basic regimes is under consideration. In the low chamber density regime ( $\lesssim 0.003$ torr), ballistic or nearly-ballistic transport is used. Partial beam neutralization has been studied to offset the effects of beam stripping. In the high chamber density regime ( $~ .1$ torr), two transport modes (pinched transport and channel transport) are under investigation. Both involve focusing the beam outside the chamber then transporting it at small radius $(\approx 2 \mathrm{~mm})$. Both high chamber density modes relax the constraints on the beam quality needed from the accelerator which will reduce the driver cost and the cost of electricity.
\end{abstract}

\section{Introduction}

Transporting the heavy ion beams from the accelerator to the target is extremely important for a successful heavy ion fusion (HIF) reactor. Furthermore, chamber

${ }^{*}$ Work performed under the auspices of the United States Department of Energy by the Lawrence Livermore National Laboratory under contract number W-7405-ENG-48. 
transport is a high leverage area since improvements in chamber transport and beam focusing can significantly reduce the cost of electricity. Relaxing the requirements on the accelerator reduces the cost of the driver which directly impacts the cost of electricity; reducing the beam spot size at the target allows a larger target gain which also reduces the cost of electricity. Improvements in beam transport and final focusing will be exploited in optimizing the end-to-end HIF system.

In the U. S. Heavy Ion Fusion program, two separate regimes are currently under study. The mainline approach is low chamber density, ballistic or nearly-ballistic transport. The HYLIFE-II reactor[1] uses a low density chamber with a pressure of a few millitorr. Even at the low density of the HYLIFE-II chamber $(\approx .003$ torr $)$, partial beam neutralization is needed to overcome the effects of beam stripping. Simulations including beam stripping and partial neutralization will be discussed in section (4). Low density transport is the most conservative option, but puts strict. requirements on the beam quality out of the accelerator.

In the high chamber density ( $\approx .1-1$ torr) regime, two approaches are being studied. The first is a "minifocus" with self-pinched transport. In the minifocus, the beam goes through the focusing system before it is drift compressed. The beam is then transported while drift compressed at small radius $(\approx 2 \mathrm{~mm})$ over about 400 meters. This system reduces the requirements on the beam quality from the accelerator and reduces the size of the holes in the reactor chamber wall. The questions that remain are whether the pinch will form and whether the pinch will be stable.

A beam can also be transported for shorter distances at small radius using a preformed current channel to provide the focusing fields. This mode does not require a self-pinch. An experiment to study a plasma lens for focusing followed by current channel guided transport is underway. This mode also reduces the requirements on the beam quality and the size of the holes in the chamber wall. The questions remaining center around the stability and interaction of the current channels in the chamber.

\section{Total Beam Spot Size at the Target}

In evaluating the different transport modes, it is useful to consider the different effects which lead to the final beam spot size at the target. The total beam spot size is influenced by the beam's space-charge and emittance, chromatic aberrations in the final focusing system, and errors in aiming the beams at the target. These sources 
are roughly independent and add in quadrature[2]

$$
\left(r_{\text {target }}\right)^{2} \approx\left(r_{\text {space charge +emittance }}\right)^{2}+\left(r_{\text {chromatic aberrations }}\right)^{2}+\left(r_{\text {aiming }}\right)^{2}
$$

where $r_{\text {target }}$ is the final spot size at the target and $r_{x}$ is the spot size from effect $x$. For high target gain, our goal is a total spot size $\leq 3 \mathrm{~mm}$.

In the HYLIFE-II reactor, the targets are injected into the chamber at a rate of 6 per second using a gas gun. Petzoldt estimates the spot size due to errors in aiming the beams at the target is $.4 \mathrm{~mm}[3]$. This estimate takes into account translational positioning errors and rotations of the target.

Chromatic aberrations occur in the final focusing system because particles with different longitudinal momenta are focused at different distances. This causes a radial spread in the particles at target which is proportional to $\delta p / p$, the longitudinal momentum spread divided by the longitudinal momentum. For a focusing system of four thin lenses, single particle calculations (neglecting space-charge) show that $r_{\text {chromatic aberrations }}=8 F \theta \delta p / p$, where $F$ is the focal distance, and $\theta$ is the half convergence angle of the beam. Including space-charge reduces the chromatic aberrations by about a factor of $3 / 4$, so

$$
r_{\text {chromatic aberrations }} \approx 6 F \theta \frac{\delta p}{p} .
$$

For a ballistic focusing system, $F$ is set by the reactor geometry and typically has a value of 5 meters. The convergence angle, $\theta$ is generally limited to about 15 mrad to avoid geometric aberrations. It is possible to use larger angles and correct the geometric aberrations using an octupole correction[4]. A larger convergence angle results in a larger aperture for the final focusing magnets, however, which increases the cost of those magnets. For the purposes of these calculations, we will keep $\theta \approx$ $15 \mathrm{mrad}$.

Limiting the spot size from chromatic aberrations to 1-1.5 mm sets a limit on the allowable momentum spread in the final focusing system. Using $F=5 \mathrm{~m}$ and $\theta=15$ $\mathrm{mrad}$, we find $\delta p / p \leq 2.2-3.3 \times 10^{-3}$ in the final focusing system. In the conventional HIF driver scenario, the beam is drift compressed by a factor of 10 or more between the end of the accelerator and the final focusing system. This drift compression increases the longitudinal momentum spread and as a result, $\delta p / p \leq 2.2-3.3 \times 10^{-4}$ at the end of the accelerator if the beam is to be drift compressed by a factor of 10 prior to focusing.

If the spot size from aiming errors of $.4 \mathrm{~mm}$, and the spot size from chromatic aberrations of $1.5 \mathrm{~mm}$, then $2.57 \mathrm{~mm}$ are left for space-charge and emittance for a 
total spot of $3 \mathrm{~mm}$. An optimization needs be done to weigh the relative costs of each of the spot size contributions. We need to assess the cost of increasing $\delta p / p$ at the expense of beam emittance, for example.

The beam spot size from space-charge and emittance can be estimated using the envelope equation

$$
a^{\prime \prime}=\frac{K}{a}+\frac{\epsilon^{2}}{a^{3}}
$$

where ' indicates a derivative with respect to $z, K$ is the perveance, $\epsilon$ is the unnormalized emittance, and $a$ is the radius. Multiplying by $a^{\prime}$ and integrating gives

$$
\left(a_{f}^{\prime}\right)^{2}-\left(a_{0}^{\prime}\right)^{2}=2 K \ln \left(\frac{a_{f}}{a_{0}}\right)-\epsilon^{2}\left(\frac{1}{a_{f}^{2}}-\frac{1}{a_{0}^{2}}\right)
$$

where 0 and $f$ denote the initial and final values. At the beam waist $a_{f}=r_{s}$, the spot size due to space charge and emittance, and $a_{f}^{\prime}=0$. At the entrance, $a_{0}^{\prime}=\theta$, the half convergence angle and $a_{0} \approx F \theta$, where $F$ is the focal length. Using these substitutions and assuming $a_{0} \gg r_{s}$, equation (4) becomes

$$
\theta^{2}=2 K \ln \left(\frac{a_{0}}{r_{s}}\right)+\frac{\epsilon^{2}}{r_{s}^{2}}
$$

In the absence of space-charge (i.e. a perfectly neutralized beam), the spot size due to emittance is given by

$$
r_{\text {emittance }}=\frac{\epsilon}{\theta}
$$

If the spot size from emittance is $1 \mathrm{~mm}$ and $\theta=15 \mathrm{mrad}$, then transverse beam emittance is restricted to $\epsilon \leq 15 \mathrm{~mm}$-mrad.

Using equation (5), we can estimate the maximum beam perveance allowed for a given spot size from space-charge and emittance. Using $\theta=15 \mathrm{mrad}, a_{0}=7.5$ $\mathrm{cm}, r_{s}=2.5 \mathrm{~mm}, \epsilon=15 \mathrm{~mm}$-mrad, equation (5) gives a maximum perveance of $K=2.8 \times 10^{-5}$. The perveance is related to the beam current by

$$
K=\frac{2 Z}{(\gamma \beta)^{3} A} \frac{I_{b}}{I_{0}}
$$

where $Z$ is the ion charge state, $\beta$ is the beam velocity over the speed of light, $\gamma$ is the Lorentz factor, $I_{b}$ is the beam current, $A$ is the ion mass in units of amu, and $I_{0}=m_{\mathrm{amu}} c^{3} / e=31 \mathrm{MA}$. For $K=2.8 \times 10^{-5}$, this leads to a maximum current per beam of $2.3 \mathrm{kA}$ for a mass 200 ion and $1.6 \mathrm{kA}$ for a mass 135 ion, both at $(\beta \gamma)=.3$. Neutralization is needed if the current per beam is larger than these values. 


\section{Ballistic Transport in Near Vacuum}

If the chamber pressure is low enough to avoid beam stripping, a beam of $10 \mathrm{GeV}$, singly charged, heavy (mass $\approx 200 \mathrm{amu}$ ) ions can be ballistically transported with a reasonable spot size. The target requires a main pulse with $4 \mathrm{MJ}$ of energy in $10 \mathrm{nsec}$ (an additional $1 \mathrm{MJ}$ is carried by a low power prepulse). The total current necessary in the main pulse is $4 \mathrm{MJ} /(10 \mathrm{GeV} \times 10 \mathrm{nsec})=40 \mathrm{kA}$. Without neutralization, the maximum current per beam found in section (2) was $2.3 \mathrm{kA}$, so 17 beams are needed for the main pulse. This provides a reasonable, conservative scenario for transporting the beam to the target.

Beam stripping is an issue, however. Estimates of the cross section $[5,6,7]$ for stripping the beam ions by the background gas in HYLIFE-II $\left(\mathrm{BeF}_{2}\right)$ range from $1.3-4 \times 10^{-16} \mathrm{~cm}^{2}$. Stripping only about $1 \%$ of the beam ions requires a chamber density $\sim 10^{11} \mathrm{~cm}^{-3}$. This density is down by two orders of magnitude from the chamber density in the HYLIFE-II reactor $\left(\approx 5 \times 10^{13} \mathrm{~cm}^{-3}\right)$. Thus, purely ballistic transport puts an undesirable restriction on the chamber density.

\section{Low Density, Nearly-Ballistic Transport}

At the density of the HYLIFE-II chamber, the stripping mean-free-path is .5-1.5 meters. In the HYLIFE-II design, the chamber radius from "first wall" to the target is 3 meters, so the beam will strip 2-6 times during chamber transport. Simulations with the BICrz code show that most of the stripped electrons tend to stay with the beam. However, in the higher charge state, the ions respond more strongly to the electric fields and the spot size increases. Simulations with stripping mean-free-path of 1.2 meters in a 3 meter chamber showed an increase in the beam spot size from $2.6 \mathrm{~mm}$ (vacuum transport) to $8 \mathrm{~mm}$. This is an unacceptably large spot and partial beam neutralization must be used to offset this increase.

While beam stripping makes chamber propagation more difficult, ionization of the background gas by the beam ions can partially neutralize the beam and aid transport. Cross sections for collisional ionization of $\mathrm{BeF}_{2}$ by the beam have a larger uncertainty than stripping cross sections because calculating molecular cross sections is more difficult than calculating atomic cross sections. Estimates of the mean-freepath for ionizing the background gas range from 0.7 to 25 meters.

Simulations show that including a stripping mean-free-path of 1.2 meters and a gas ionization mean-free-path of 3.0 meters reduced the spot size from $\& \mathrm{~mm}$ to $5.4 \mathrm{~mm}$. 
Neutralization by gas ionization occurs "for free" since we do not have to add anything to the reactor for it to occur; however, gas ionization neutralizes the beam slowly so radial velocities develop before neutralization occurs. The solid curve in figure (1) shows the neutralization fraction as a function of distance from the chamber wall for a simulation without beam stripping. The beam is more than $70 \%$ neutralized, but it takes about 2.5 meters of propagation distance to reach this level of neutralization. To get a smaller spot, additional neutralization is needed.

\subsection{Neutralization Using a Preformed Plasma Annulus}

Neutralization of an ion beam is more difficult than neutralizing an electron beam. When an electron beam passes through a plasma, the plasma electrons are moved out of the beam path and the beam is neutralized by the immobile ions. For an ion beam, electrons must be pulled in from outside the beam path in order to reduce the net charge.

One method for neutralizing the beam quickly is to create a preformed plasma in the chamber before the beam enters. Simulations used a small $(.3$ meter $=40 \%$ of the beam length) annulus of plasma just inside the chamber entrance. The dashed curve in figure (1) shows that the beam is neutralized quickly and was more than $90 \%$ neutralized while inside the annulus. As expected, neutralization reduces the beam spot size at the target. In a simulation with a stripping mean-free-path of 1.2 meters and a plasma annulus, the final spot was $3.5 \mathrm{~mm}$. This is a significant decrease over the $8 \mathrm{~mm}$ spot found without neutralization, but is not as good as the pure vacuum transport result of $2.6 \mathrm{~mm}$.

The dashed curve in figure (1) shows that while the beam is well neutralized near the chamber entrance, it does not remain well neutralized. The electrons pulled in from the plasma annulus are hot $\left(v_{\text {th }} \sim .3 \mathrm{c}\right)$. As the beäm compresses, the electrons do not compress as readily as the beam and the neutralization fraction falls off as the beam approaches the target. Electrons created by collisional ionization of the background gas are cooler than those pulled in from the plasma annulus and we expect the smallest spot when both collisional ionization and a plasma annulus are included. Simulations confirm this and the spot is reduced from $3.5 \mathrm{~mm}$ to $3.0 \mathrm{~mm}$ when a gas ionization mean-free-path of 3 meters is added to the simulation. 


\subsection{Neutralization Using a Plasma Column}

In the case of the plasma annulus, neutralization was quite good $(>90 \%)$ inside the annulus, but the neutralizing electrons did not compress with the beam. As a result, neutralization got worse as the beam got closer to the target. One method to remedy this problem is to put the plasma throughout the entire chamber so the beam can continually pull in new electrons as it compresses.

The plasma density required in the plasma column is not large. Simulations show that ionizing just $0.44 \%$ of the background gas $\left(n_{e}=2.5 \times 10^{11} \mathrm{~cm}^{-3}\right)$ in a cylinder

of radius $\sqrt{2} r_{\text {beam, initial was enough to eliminate the effects of beam stripping when }}$ the stripping mean-free-path was 1.2 meters. In this case, the electron density was 6 times the initial beam density. As the beam compressed, the beam density became much larger than the electron density.

One method for reducing the cost of the driver is to use a lighter, lower energy ion such as $5.3 \mathrm{GeV} \mathrm{Cs}^{+}$. The cost of using the lighter, lower energy ion is that more current is necessary to deliver the same energy to the target. For $5.3 \mathrm{GeV} \mathrm{Cs}^{+}, 75 \mathrm{kA}$ of current is needed to provide $4 \mathrm{MJ}$ of energy in the $10 \mathrm{nsec}$ main pulse. We found in section (2) that the maximum current we can transport in the chamber for a mass 135 ion without neutralization is $1.6 \mathrm{kA}$ per beam. This means we need more than 45 unneutralized cesium beams. With about $80 \%$ neutralization, we can transport the $75 \mathrm{kA}$ of cesium in 10 beams.

The low density plasma column neutralized the cesium beam quite well. In a simulation with a $7.5 \mathrm{kA}$ beam of $5.3 \mathrm{GeV} \mathrm{Cs}^{+}$ions (without beam stripping) and a low density plasma column $\left(n_{e}=2.3 \times 10^{11} \mathrm{~cm}^{-3}\right)$ produced a spot of $1.2 \mathrm{~mm}$. This simulation used a smaller emittance $(15 \mathrm{~mm}$-mrad) than was used in some of the previous cases. For the same parameters, the unneutralized beam spot was $9 \mathrm{~mm}$ and the perfectly neutralized spot (from emittance only) was $.9 \mathrm{~mm}$. Figure (2) shows the simulation particles at 3 times plus the envelope solution for the unneutralized beam (dashed curve) and the fully neutralized beam (solid curve).

Adding a beam stripping mean-free-path of 3.2 meters to the cesium beam simulation produced a spot of $2 \mathrm{~mm}$. This mean-free-path would correspond to a decrease of about a factor of two in the chamber density from the standard HYLIFE-II case.

\subsection{Remaining Issues}

Producing the plasma column in the HYLIFE-II chamber still needs to be addressed. Some methods under consideration are using a discharge, or a laser to ionize some 
of the chamber vapor. Another possible method is to use a plasma gun to create the plasma and inject it in the chamber. Any equipment used to create the plasma (lenses, insulators, etc.) must be protected from the blast. This work is in progress.

Experiments are needed to verify the results of the simulations. We believe that experiments to study beam neutralization can be done using existing facilities at LBNL or SABRE at Sandia National Laboratory.

Beam neutralization thus far has concentrated on "passive" neutralization using plasmas in the chamber. "Active" neutralization by co-injecting an electron beam along with the ion beam is another possibility for beam neutralization. Further study in this area is needed.

\section{Autoneutralizing Targets}

As the beams approach the target, they begin to overlap and the space-charge forces become very large. A method for neutralizing the beams in the last 10-15 centimeters has been proposed using an "autoneutralizing" target [8, 9]. The autoneutralizing target is a conventional, two-sided indirect drive target which has 10-15 cm cylinders attached near the radiators (see figure (3)). The beam passes through a submicron plastic film at the end of these cylinders. The beam's space-charge draws electrons from the film which co-move with the beam ions and neutralize the beam.

The autoneutralization takes place in the last few centimeters of chamber transport where the beams overlap and the space-charge forces are the largest. In addition, as the target starts to radiate, photons can further ionize the beam ions close to the target which causes them to respond more strongly to the electric fields. Autoneutralization could also be used to supplement other low density neutralization schemes. Neutralization by a plasma, for example, does not work as well near the target.

Previous simulations seem to indicate that large space-charge forces near the target are not very important, however. This was seen in simulations of beam photoionization. In these simulations, the beam ions were photoionized by radiation from the target as the target heated. These simulations showęd only $5 \%$ of the ions fell outside the desired spot even though the ion charge state was increased significantly. The photoionization had little effect on the spot size because the ionization took place close to the target and there was little time for any velocity deflections to become a spread in position[10]. The role of autoneutralizing targets is under investigation. 


\section{Pinched Transport}

In the low chamber density final focus/chamber scenario, the beam exits the accelerator and is drift compressed by a factor of 10 over a distance of about 400 meters. It then enters the final focusing system, located just outside the chamber entrance.

The "minifocus" concept reverses the order of these operations (see figure (4)). In the minifocus, the beam is focused to a small radius $(\approx 2 \mathrm{~mm})$ just after it leaves the accelerator. The beam is fully stripped by a foil when it reaches its final radius. It is then drift compressed over the 400 meters while at small radius. Confinement is provided by the beam's self magnetic field. To achieve an equilibrium radius of 2 $\mathrm{mm}$, a beam of $5.3 \mathrm{GeV}, \mathrm{Cs}^{55+}$ must be $100 \%$ charge neutralized and $99 \%$ current neutralized. A .1-1 torr gas is added to the beam pipe to help with neutralization. The excess current provides a magnetic field which creates the pinch.

The minifocus/pinched transport scheme has a variety of advantages over the traditional ballistic or nearly-ballistic transport scheme. In the conventional final focusing system the focal length is determined by the reactor geometry. The reactor chamber radius cannot be much smaller than 3 meters because the walls need to be protected from the blast. In the minifocus, the focal length is no longer determined by the reactor geometry and shorter focal lengths can be used. This leads to smaller aperture magnets which should cost less than the magnets in the conventional system. In addition, the space-charge forces in the focusing magnets are smaller because the beam has not yet been drift compressed.

The most important difference in beam parameters is the amount of longitudinal momentum spread allowed in the accelerator. Since the beam is drift compressed after it is focused, the $\delta p / p$ is in the accelerator can be 10 times larger than in the conventional system. In addition, the smaller focal length allows another factor of 5-10 increase in the longitudinal momentum spread. Allowing a larger momentum spread in the accelerator means less precision is needed in the accelerating fields and the "ear" fields used to confine the beam longitudinally. A larger longitudinal momentum spread will also stabilize or partially stabilize the longitudinal instability[11].

Because the beam is at small radius before it enters the chamber, the minifocus/pinched transport scenario creates smaller holes in the chamber wall. Smaller holes lead to less debris in the beam pipes just upstream of the chamber.

The challenge in the minifocus is to transport the $2 \mathrm{~mm}$ radius beam for a distance of 400 meters. The first question that needs to be answered is whether or not the pinch will form. Particle-in-cell simulations have begun to explore this question[12]. At present, the results have not been conclusive either way. 
If the pinch forms, questions of stability still exist. Two instabilities that might affect the pinch are a kink instability and a two-stream instability. Over most of the 400 meters, the kink mode will be wall stabilized by the beam pipe. Over the last few meters in the chamber, there is no beam pipe and this instability could be a problem. The two-stream instability is another concern. Preliminary calculations show some indication of two stream instability between the electrons riding with the beam and the background gas ions[12].

\section{Channel Transport}

Another method of final focus/beam transport under investigation is a system involving a plasma lens followed by channel transport. In this scenario, the beam is drift compressed after the accelerator. A conventional final focusing magnet system does the first stage of beam focusing, but is followed by a plasma lens so that the beam reaches its final radius outside the chamber. The beam is transported at small radius through the chamber using a preformed current channel to confine the beam. Figure (5) shows a schematic layout of the reactor chamber.

Plasma lenses have been under investigation by the German Heavy Ion Laboratory GSI for several years[13]. The plasma lens uses a wall-stabilized $z$-pinch discharge to generate the magnetic field used for focusing. This generates a strong focusing field in both directions simultaneously (first order focusing) in contrast to conventional quadrupole magnets which focus in one plane and defocus in the other (a doublet is then second order focusing). For an HIF final focus, a thick adiabatic lens will be used. This lens allows a much larger beam emittance and longitudinal momentum spread than a conventional focusing system $[14,15]$.

As in the minifocus, the beam reaches its final radius outside the chamber which decouples focusing from chamber transport. The beam is then transported at small radius through the reactor chamber. This is done using a preformed current channel to provide the magnetic field which confines the beam. A laser is used to ionize four channels in the chamber. A discharge is driven in these channels by a capacitor bank close to the reactor wall. Two current channels are used to transport the beams to the two-sided target. Two additional channels provide a return path for the discharge current. The beam will be neutralized by electrons pulled in from the plasma lens so space-charge effects in the chamber should be small or nonexistent.

An experimental effort is underway at Lawrence Berkeley National Laboratory (LBNL) to study the plasma lens and channel transport $[15,16]$. One experiment 
will study the plasma lens using the LBNL $2 \mathrm{MeV}$ ESQ injector[17]. The second experiment is to explore issues surrounding the current channels. This experiment will address instabilities in the channels, the interaction of the channels with the return current paths, and the interaction of the channels with the target.

\section{Interactions Between Neighboring Beams}

Most indirect drive, HIF target designs have two radiation converters, while most transport schemes (ballistic and nearly-ballistic transport, minifocus, for example) involve more than two beams. As a result, multiple beams will be aimed at a single radiation converter and these beams will be fairly close to one another in the chamber (see figure (6)). As a result, each beam will be affected by the fields from neighboring beams and this can result in an increase in the beam spot size.

If the fields due to the neighboring beams were constant along the beam length, we could compensated for them by increasing the focusing angle slightly. The fields are not constant, however, and vary along the pulse because of variations in the beam current as a function of $z$ and the finite length of the beams. These two effects cause the field at the ends of the beam to be smaller than the field at the beam center. Since we cannot adjust the focusing angle on the timescale of the beam pulse duration ( $10 \mathrm{nsec}$ for the main pulse), the beam ends will be overfocused if the beam center is focused.

Hofmann, Hasse, and Reiser studied this problem for a cone of beams produced by an RF Linac with storage rings[18]. In the RF Linac approach to HIF, beam compression is done by a phase rotation and results in a beam with a roughly Gaussian current profile. The variation in current along with the finite length of the beams causes a large variation in the electric field between the beam center and the beam ends. Hofmann, et. al., found the increase in spot size due to the neighboring beams was tolerable for a charge state +1 beam, but scaled as the charge state squared, so that it was not acceptable for higher charge state ions.

This problem is less severe for beams produced by an induction linac. The induction linac can produce a nearly flat topped current pulse so variations in the electric field come almost exclusively from the finite length of the beams. Calculations show $\leq 5 \%$ beam loss for a "fan" of either 6 unneutralized, $4 \mathrm{kA}$ beam of $10 \mathrm{GeV} \mathrm{Pb}^{+}$ions or $70 \%$ neutralized, $7.5 \mathrm{kA}$ beam of $5.3 \mathrm{GeV} \mathrm{Cs}^{+}$ions.[19]

Complete simulations of the neighboring beams problem will require a fully 3 dimensional, electromagnetic code. Such a code is under development by J.-L. Vay 
and C. Deutsch at University of Paris-Orsay[20].

\section{Chamber Transport for Light Ions, Heavy Ions, and Middle-Weight Ions}

There has been interest in exploring the possibility of using a middle-weight ion for a combined Laboratory Microfusion Facility (LMF) and Engineering Test Facility (ETF). Three working groups will study chamber transport, acceleration, and target physics for a middle-weight ion. The chamber transport working group held a workshop to study chamber transport for a "common" ion driver in September 1994 at Sandia National Laboratory[21].

For chamber transport, there did not seem to be any reason to favor a middleweight ion. However, the workshop reinforced the commonality that exists between light ion fusion (LIF) chamber transport and HIF chamber transport. Collaborations between these two programs already exists and there was enthusiasm for increasing this effort. In particular, some transport schemes, such as self-pinched transport, look attractive for LIF, HIF and a common ion. Experiments to benefit both programs can be done on existing and proposed machines such as SABRE at SNL, and ILSE/Elise at LBNL. In addition, collaborations on code development and cross utilization of codes will be beneficial to both programs.

\section{Conclusions}

Several methods for chamber transport/final focus are currently under investigation. In the low chamber density regime, the goal is to understand the differences between pure vacuum, ballistic transport, which is impractical in a reactor, and low density, nearly-ballistic transport. The conclusion has been that beam stripping needs careful attention, but simulations show that partial beam neutralization can overcome the limitations of beam stripping. Neutralization of higher current beams of lower mass, lower energy ions looks promising. The lower energy beam would reduce the cost of the driver, and ultimately, the cost of electricity. The HYLIFE-II reactor design is based on low chamber density, ballistic or nearly-ballistic transport.

Higher chamber densities provide an opportunity to increase the allowable emittance and/or longitudinal momentum spread in the beam. This makes building the accelerator easier and less expensive which reduces the cost of electricity. Both pinched 
transport and channel transport modes are currently under study. These transport modes are also of great interest to the Light Ion Fusion program and provides an opportunity for collaboration in both experiments and simulations.

\section{References}

[1] R. W. Moir, R. L. Bieri, X. M. Chen, T. J. Dolan, M. A. Hoffman, P. A. House, R. L. Leber, J. D. Lee, Y. T. Lee J. C. Schrock, M. T. Tobin, W. H. Williams, Fusion Technology, 25, 5 (1994).

[2] E. P. Lee, Heavy Ion Inertial Fusion, M. Reiser, T. Godlove, and R. Bangerter, Eds., AIP Conference Proceedings, American Institute of Physics, New York, NY, p. 461 (1986).

[3] R. W. Petzoldt, PhD Thesis, University of California at Davis, 1995.

[4] D. D.-M. Ho, I. Haber, K. R. Crandall, S. T. Brandon, Particle Accelerators, 36, 141 (1991).

[5] N. Barboza, U.C. Berkeley, Private Communication (1994).

[6] W. R. Meier, et. al.; "Osiris and Sombrero Inertial Confinement Fusion Power Plant Designs," WJSA-92-01, DOE/ER/54100-1 (1992).

[7] B. Badger, et. al., "HIBALL-2, An Improved Conceptual Heavy Ion Beam Driven Fusion Reactor Study," UWFDM-625, U. of Wisconsin (1984).

[8] D. D.-M. Ho, S. T. Brandon, submitted to Nuclear Fusion, 1995.

[9] D. D.-M. Ho, these proceedings.

[10] A. B. Langdon, Particle Accelerators, 37-38, 175 (1992).

[11] E. P. Lee, Il Nuovo Cimento, 106, 1679 (1993).

[12] K. D. Hahn, these proceedings.

[13] E. Boggasch, A. Tauschwitz, H. Wahl, K.-G. Dietrich, D. H. H. Hoffmann, W. Laux, M. Stetter, R. Tkotz, Appl. Phys. Letters, 60, 2475 (1992). 
[14] P. Chen, K. Oide, A. M. Sessler, S. S. Yu, Phys. Rev. Letters, 64, 1231 (1990).

[15] A. Tauschwitz, S. S. Yu, S. Eylon, L. Reginato, W. Leemans, J. O. Rasmussen, R. O. Bangerter, Proceedings of the 1995 Particle Accelerator Conference, Dallas, TX, May 1-5, 1995.

[16] A. Tauschwitz, these proceedings.

[17] S. S. Yu, these proceedings.

[18] I. Hofmann, R. W. Hasse, M. Reiser, J. Appl. Phys., 73, 7061 (1993).

[19]. D. A. Callahan, UCRL-JC-121132, submitted to Appl. Phys. Letters, 1995.

[20] J.-L. Vay, these proceedings.

[21] C. L. Olson, E. P. Lee, A. B. Langdon, "Workshop on Transport for a Common Ion Driver," Sandia National Laboratory, Albuquerque, NM, September 20-21, 1994 (SNL Document SAND95-0116, UC-712). 
Figure 1: Neutralization fraction as a function of distance from the chamber entrance for neutralization using collisonal ionization of the background gas (solid curve) and neutralization using a preformed plasma annulus in the chamber (dashed curve)

Figure 2: A particle-in-cell simulation shows that a 7500 Ampere beam of $5.3 \mathrm{GeV}$ $\mathrm{Cs}^{+}$ions is well neutralized by a low density plasma column. The dashed curve shows the envelope equation with no neutralization while the solid curve shows the envelope solution with perfect neutralization

Figure 3: The beams pass through a thin plastic film and are neutralized $10-15 \mathrm{~cm}$ from the radiator in the autoneutralizing target. This provides neutralization very close to the target where the multiple beams overlap and the space-charge forces are the largest.

Figure 4: In the minifocus concept, the beam is focused just downstream of the accelerator, then is drift compressed and transporled at small radius $(\approx 2 \mathrm{~mm})$ to the reactor chamber. The beam is confined by a self-pinch.

Figure 5: A schematic of a plasma lens/channel transport reactor chamber. Only one side is shown in detail. The beams are focused just outside the chamber by an adiabatic plasma lens, then transported at small radius through the chamber to the target. A laser is used to preionize four channels which are driven by a discharge to provide the fields to confine the beam. Two channels are used for beam transport while the other two provide a path for the return current.

Figure 6: One option for multiple beam transport in the HYLIFE-II reactor is to place 6 beams in a "fan" aimed at one radiation converter. 


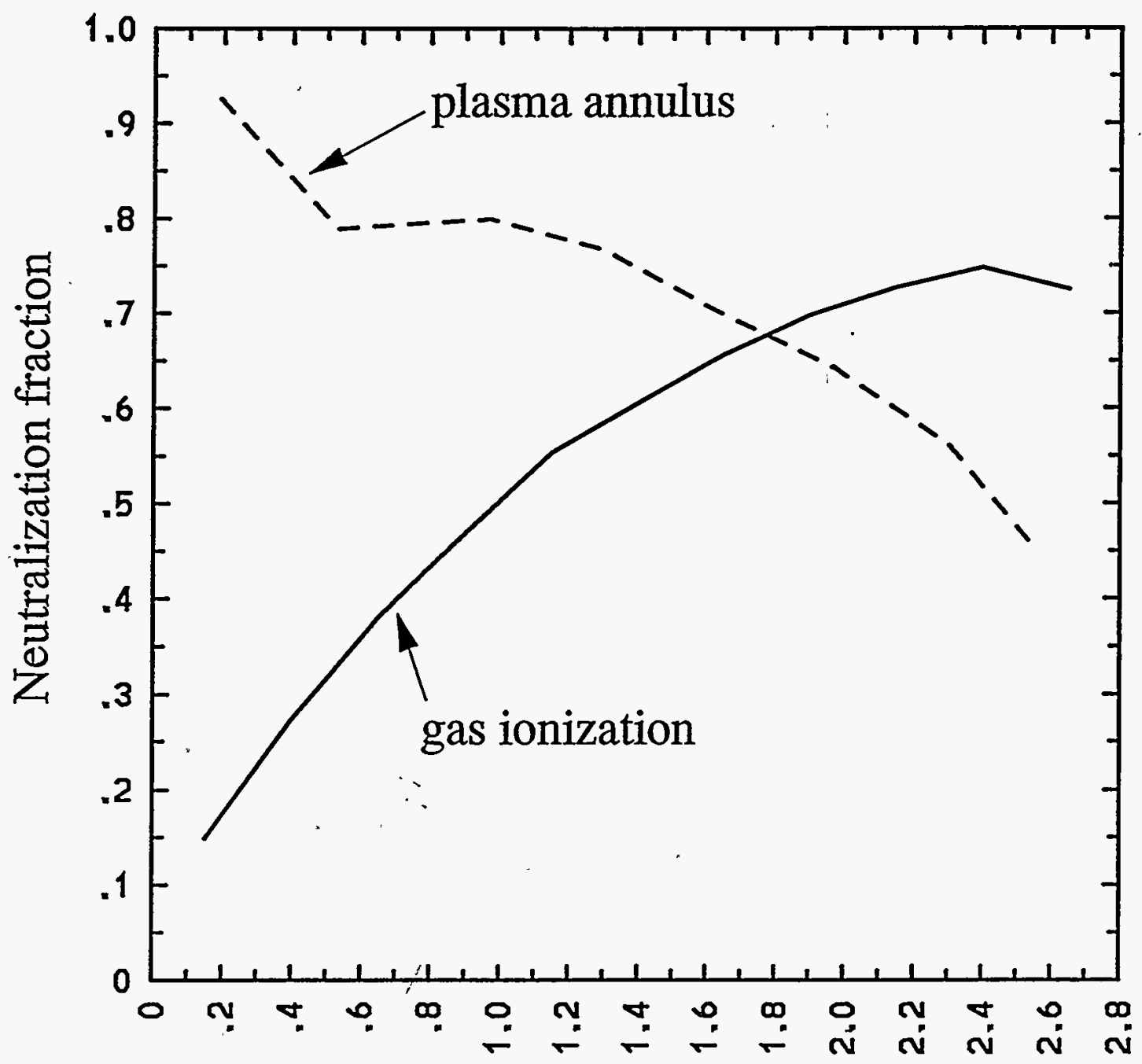

Distance from chamber entrance (m)

fig 1

Cellnham N.lan 


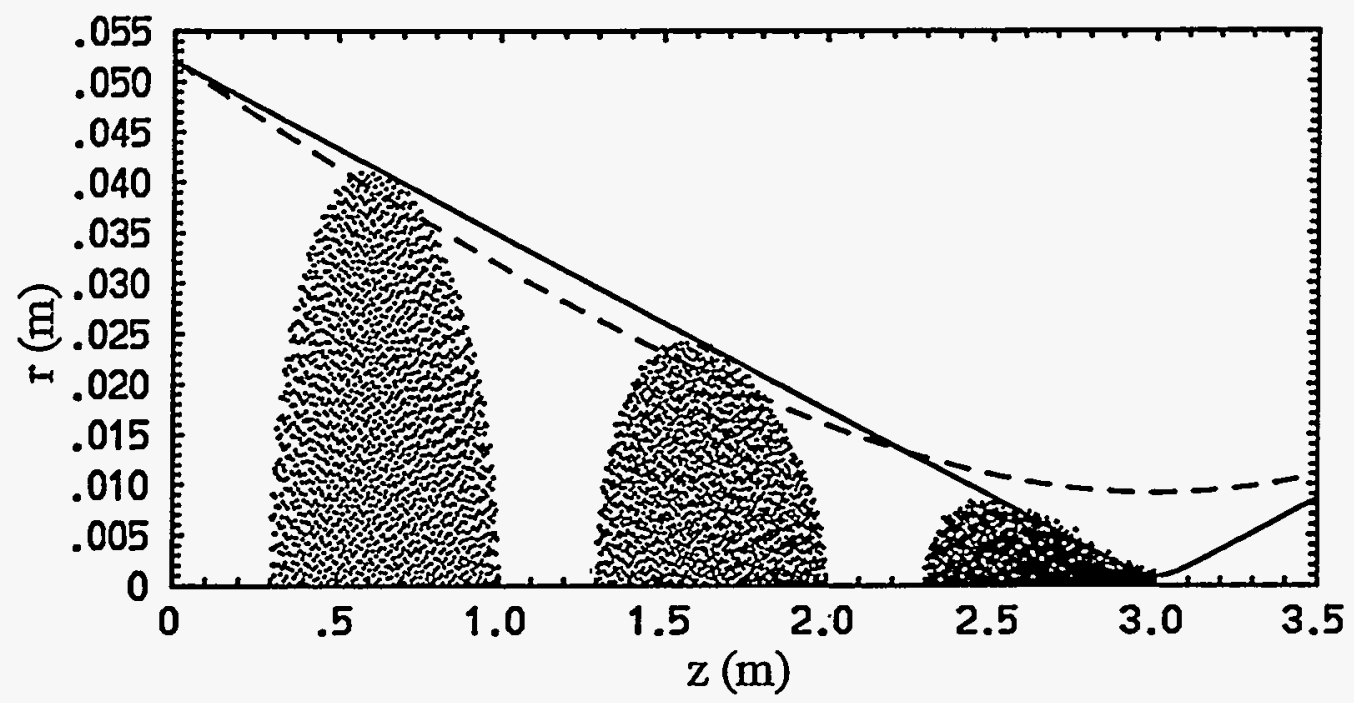

fig 2

Callahan Dolms $A$ 


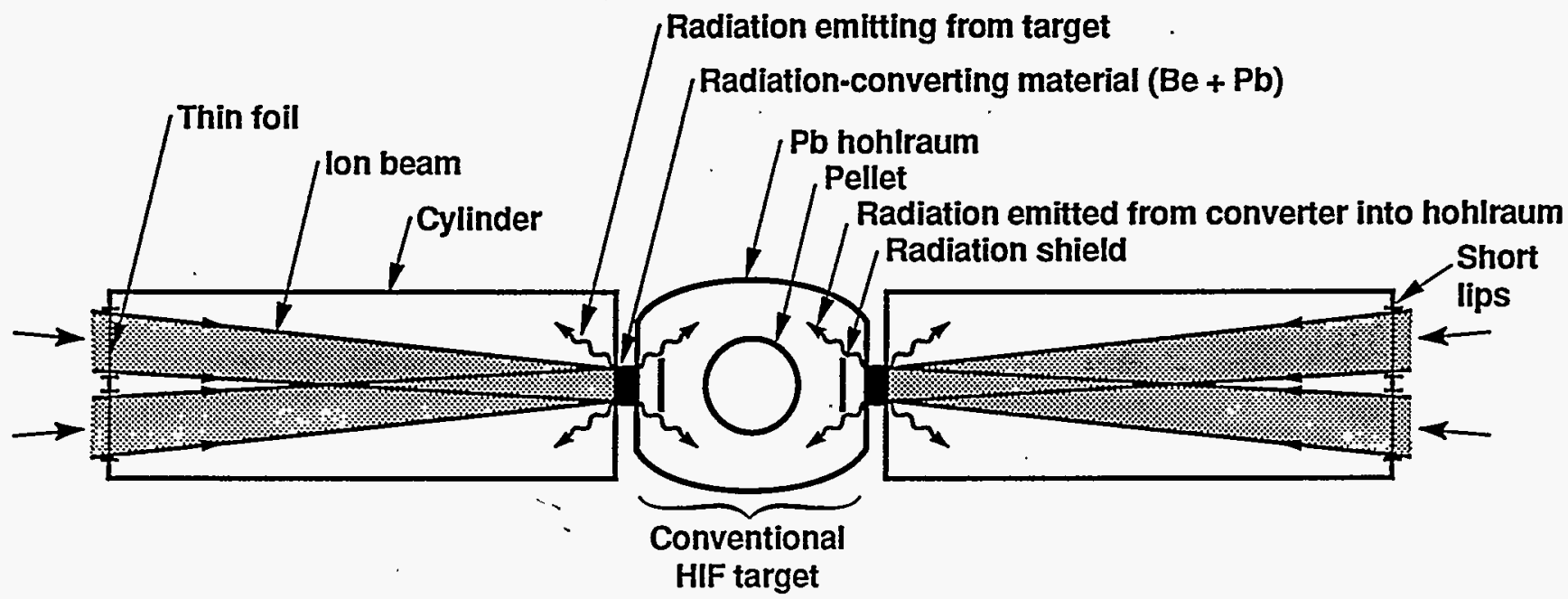

fig 3

Crillolinom Dolere $\Delta$. 


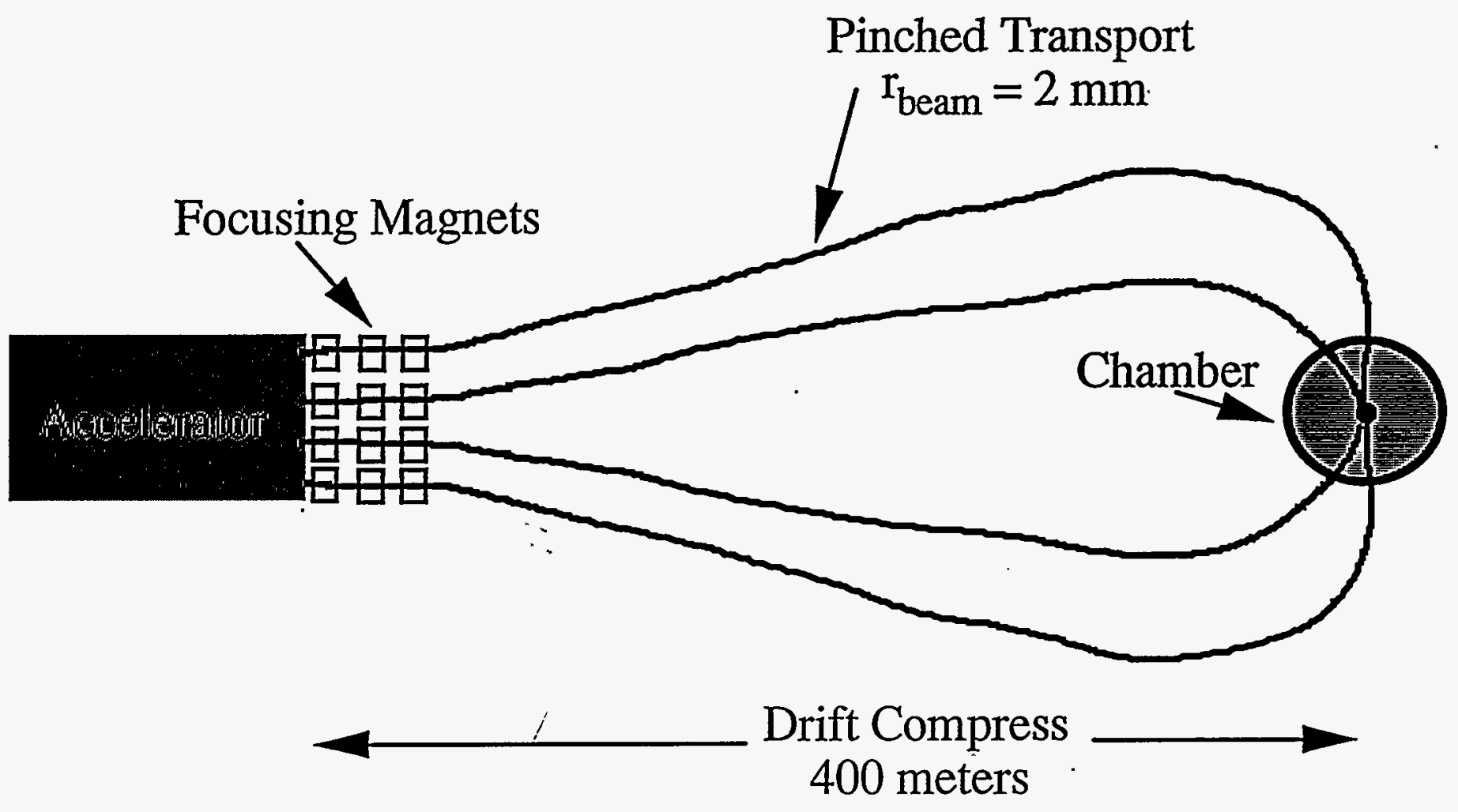

Callahan, Debra A. fig 4 


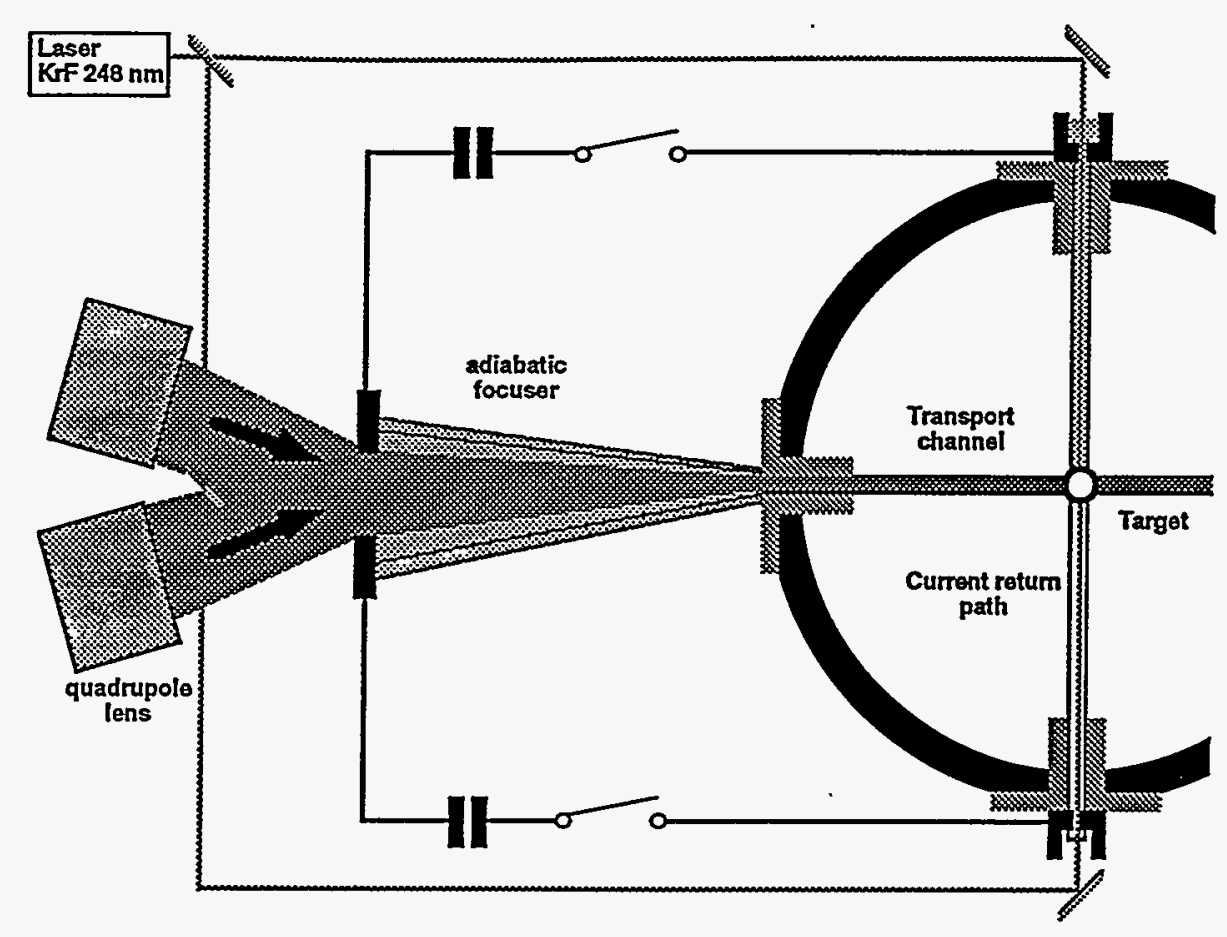

Callahan, Debra A

fig 5 


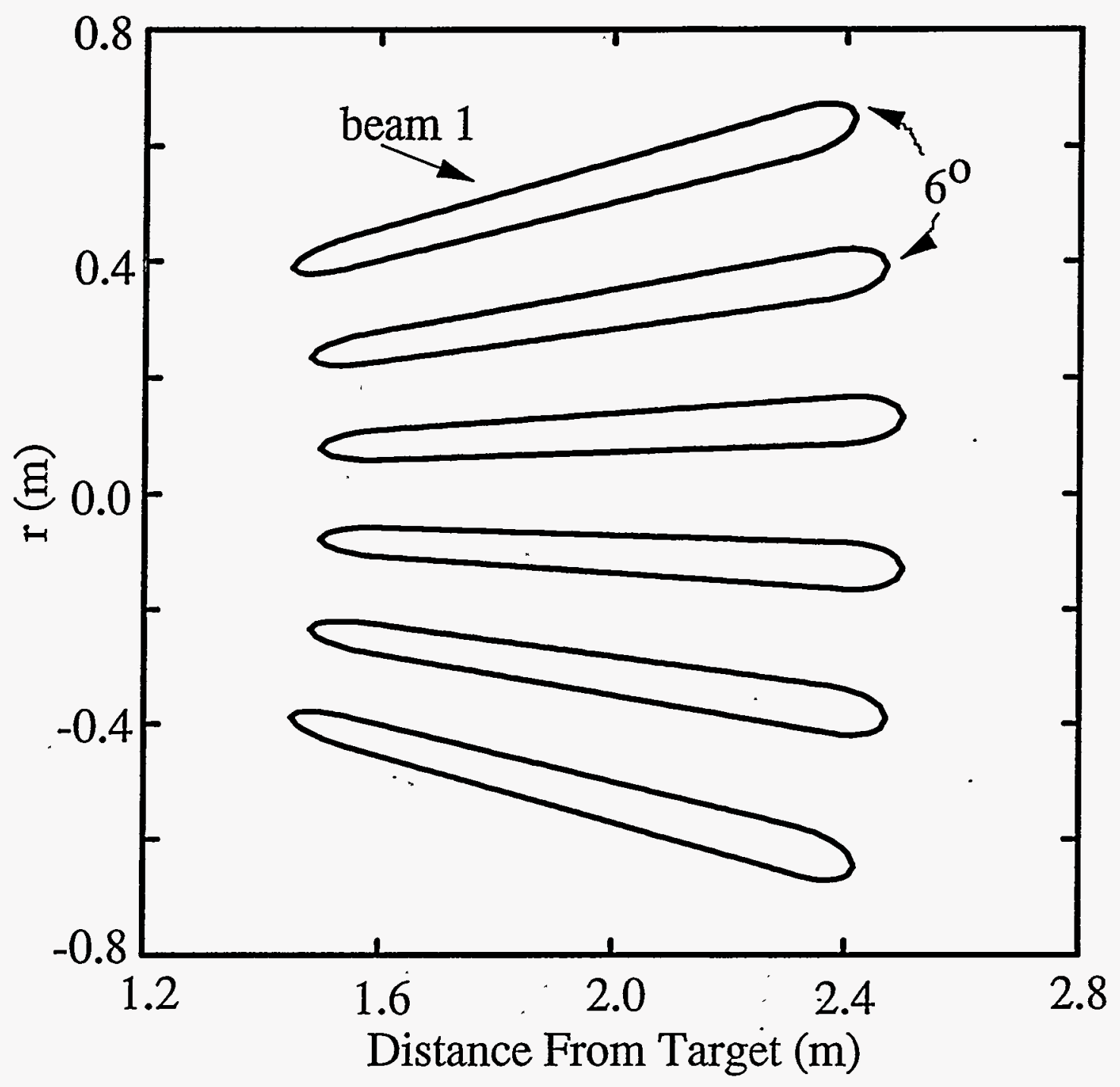

Callahan, Debra A. fig 6 
Technical Information Dcpartment . Lawrence Livermore National Laboratory University of California - Livermore, California 9455

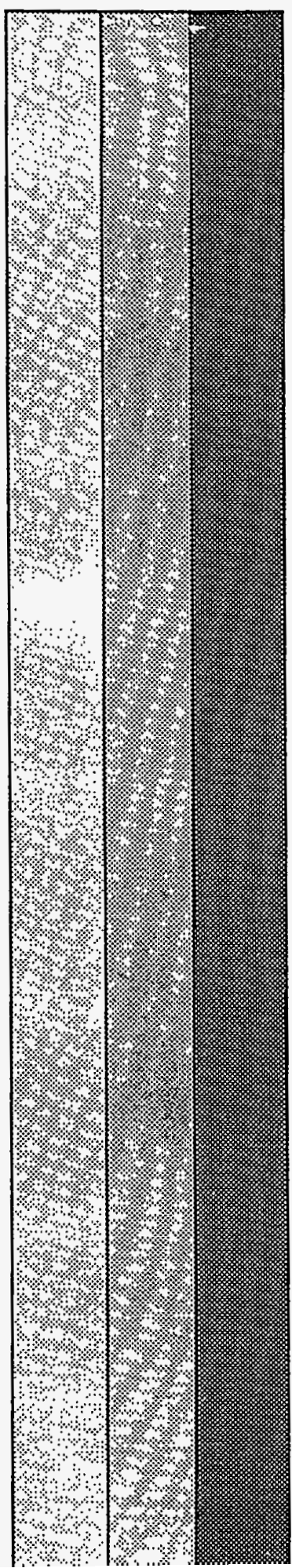

